\title{
19 RESVERATROL IMPROVES INSULIN RESISTANCE WITH ANTI-INFLAMMATORY AND "BROWNING" EFFECTS IN ADIPOSE TISSUE OF OVERWEIGHT HUMANS
}

R Gospin, ${ }^{1}$ O Sandu, ${ }^{1}$ K Gambina, ${ }^{1}$ A Tiwari, ${ }^{1}$ M Bonkowski, ${ }^{2}$ M Hawkins ${ }^{1}$. ${ }^{1}$ Endocrinology, Albert Einstein College of Medicine, Bronx, NY, United States;

${ }^{2}$ Harvard Medical School, Boston, MA, United States

\subsection{6/jim-2016-000080.35}

Purpose of Study Resveratrol is a plant-derived polyphenol whose beneficial metabolic effects in rodents include improved insulin sensitivity, reduced inflammation, and increased muscle mitochondrial biogenesis. We set out to confirm these findings in insulin resistant human subjects, by examining the effects of resveratrol on insulin sensitivity, muscle mitochondria, and adipose inflammation.

Methods Used Specifically, resveratrol $2 \mathrm{gm} /$ day (RV) or placebo (PL) were administered for 28 days in a randomized, double-blinded fashion to $\mathrm{n}=21$ non-diabetic subjects $\quad\left(17 \mathrm{M} ; \quad\right.$ Age $=52 \pm 2 ; \quad$ BMI $=31.9 \pm 0.9 \mathrm{~kg} / \mathrm{m}^{2}$; HOMA-IR $=3.9 \pm 0.2$ ). All subjects participated in 6 -hour, stepped euglycemic hyperinsulinemic $\left(30\right.$ and $80 \mathrm{mU} / \mathrm{m}^{2}$. min) 'pancreatic clamp' studies to assess hepatic and peripheral insulin sensitivity, with biopsies of vastus lateralis muscle and subcutaneous abdominal adipose tissue, before and after RV and PL. Muscle mitochondria were analyzed for quantity, size, area in a field, and the $\%$ area covered, using electron microscopy with Volocity image analysis.

Summary of Results RV induced a 22\% $(p=0.035)$ increase in glucose uptake, but did not affect glucose production. There were no changes in quantity $(p=0.829)$ or percent area $(p=0.897)$ of muscle mitochondria. There were no changes in basal or resting energy expenditure (Kcal/day) or respiratory quotient, as assessed by indirect calorimetry (Deltatrac), or in muscle strength in these healthy middle-aged subjects. However, RV reduced adipose tissue inflammation, with decreased expression of the pro-inflammatory cytokines TNF $\alpha$ and IL6 in whole fat (by $68 \%$ and $52 \%, \mathrm{p}<0.05$ ), and of IL6 and PAI-1 in adipose macrophages (by $50 \%$ and $40 \%, \mathrm{p}<0.05$ ). Adiponectin expression in whole fat increased by $53 \%$ with RV. Furthermore, we observed increased expression of genes associated with 'browning' of adipose tissue, including UCP1 and PGC- $1 \alpha \quad(46.6 \%$ and $34.9 \%$ increases, respectively). 
Conclusions Thus, while improved insulin sensitivity was not accompanied by changes in size or number of muscle mitochondria, anti-inflammatory and 'browning' effects in adipose tissue could contribute to resveratrol's favorable metabolic effects in insulin resistant humans. 